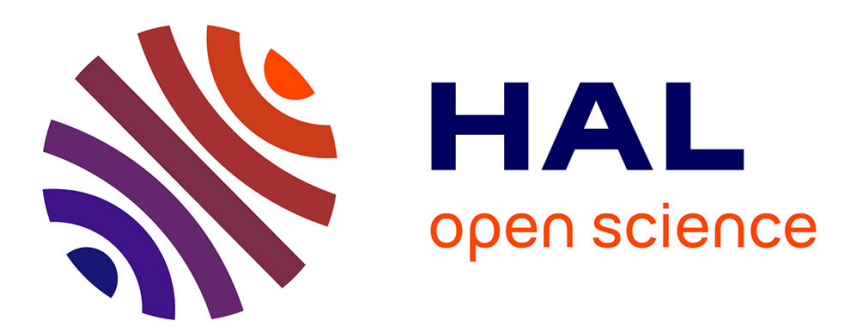

\title{
Henri Fayol et la théorie du chef d'entreprise: une nouvelle figure de l'autorité au tournant du XXe siècle
}

Armand Hatchuel, Blanche Segrestin

\section{To cite this version:}

Armand Hatchuel, Blanche Segrestin. Henri Fayol et la théorie du chef d'entreprise: une nouvelle figure de l'autorité au tournant du XXe siècle. Entreprises et Histoire, 2016, 2 (83), pp.108 - 108. 10.3917/eh.083.0108. hal-01501332

HAL Id: hal-01501332

https://hal-mines-paristech.archives-ouvertes.fr/hal-01501332

Submitted on 9 Jun 2017

HAL is a multi-disciplinary open access archive for the deposit and dissemination of scientific research documents, whether they are published or not. The documents may come from teaching and research institutions in France or abroad, or from public or private research centers.
L'archive ouverte pluridisciplinaire HAL, est destinée au dépôt et à la diffusion de documents scientifiques de niveau recherche, publiés ou non, émanant des établissements d'enseignement et de recherche français ou étrangers, des laboratoires publics ou privés. 


\section{Henri Fayol et la théorie du chef d'entreprise \\ Une nouvelle figure de l'autorité au tournant du 20 è siècle.}

A. Hatchuel, B. Segrestin

\section{Résumé}

Avant d'écrire son célèbre Traité, Henri Fayol fut un dirigeant, innovateur et savant, qui considérait la recherche scientifique comme une responsabilité majeure du chef d'entreprise. Or, devenue le moteur du monde industriel, la science créait un rapport inédit au futur : elle forçait à agir collectivement dans l'inconnu. Ce constat nous semble éclairer la doctrine administrative de Fayol sous un jour nouveau. Il explique pourquoi Fayol recourt à des notions originales (prévoyance, inconnu, programme d'action, perfectionnement, constitution du corps social) dont la portée théorique et politique a été sous-estimée. Elles permettent à Fayol de penser un nouveau chef d'entreprise sans référence à la langue des affaires, et à l'économie politique de son temps. C'est dans l'héritage des lumières et dans la philosophie politique et sociale qu'il puise pour élaborer un modèle que nous appelons "créatif/politique » de l'autorité du dirigeant d'entreprise. Ce modèle marque un tournant majeur même si le message Fayolien a été ensuite banalisé par ses traducteurs ou ses commentateurs. Un siècle après, retrouver Fayol, offre une ressource théorique précieuse pour penser des alternatives à la conception financiarisée de l'entreprise.

Armand Hatchuel est professeur à MinesParistech-PSL Research University. Il co-dirige la Chaire de théorie et méthodes de la conception innovante. Derniers ouvrages publiés (en collaboration) Refonder l'entreprise (Seuil 2011), Théorie, méthodes et organisation de la conception (Presses de Mines 2014), La société à Objet social étendu (Presses des Mines 2015).

Blanche Ségrestin est professeur à MinesParisTech-PSL Research university et directrice adjointe-du centre de Gestion Scientifique. Elle dirige la Chaire de Théorie de l'entreprise. Derniers ouvrages publiés (en collaboration) Refonder l'entreprise (Seuil 2011), La société à Objet social étendu (Presses des Mines 2015), L'entreprise, point aveugle des savoirs (Sciences Humaines 2015). 


\section{Pourquoi relire ou lire Fayol, un siècle après ?}

Il y a un siècle, paraissait la première publication du traité " d'Administration industrielle et générale ${ }^{1}$ (Fayol 1916-17, le "Traité » dans la suite). Henri Fayol est ensuite rentré dans I'histoire comme le père d'une doctrine administrative fondée sur des principes rigides et universels, rationaliste et qui fut souvent réduite à quelques formules dans la littérature (Mintzberg 1976, Lamond 2004). Récemment, plusieurs réévaluations de son œuvre ont été engagées (Chambers 1975, Peaucelle 2003 a, 2003b ; Pryor et al. 2010). Elles montrent que Fayol avait prévu certaines tendances du management au 20 è siècle (Fells 2000; Wren 2001 ; Parker et al. 2005 ; Yoo et al. 2006). Elles soulignent son rôle précurseur dans l'invention des "outils de gestion" (Peaucelle 2003). Elles examinent les relations entre ses recommandations et son action (Reid 1995 a et b). Mais dans l'ensemble, ces travaux ne remettent pas en cause la lecture traditionnelle du Traité, telle qu'elle a été fixée, avant la seconde guerre mondiale, notamment aux USA (Urwick 1949, Wren 2001 ; Wren et al. 2002).

Dans cet article, nous présentons les éléments d'un réexamen critique du Traité. Trois éclairages convergents autorisent à lire Fayol autrement $:$ i) les figures traditionnelles du chef dont il a voulu s'écarter ; ii) le nouveau contexte industriel et scientifique dans lequel il était plongé ; iii) les indications de Fayol lui-même affirmant explicitement dans un texte publié mais peu étudié ${ }^{2}$ qu'un message $^{\prime}$ majeur de son Traité était le nécessaire -et novateur- rapprochement de la science et l'industrie :

"Et ce n'est pas la tâche la moins difficile du chef d'entreprise que de conjuguer les efforts des savants et des praticiens. Il y a de nombreux obstacles à surmonter : je l'ai montré dans mon ouvrage sur l'Administration industrielle et générale; mais en même temps j'ai proclamé l'indispensable nécessité pour l'industriel d'organiser et de réussir la collaboration de la science avec le monde des affaires. Cette idée pleine de promesses et qui vient maintenant à l'honneur m'est chère depuis bien longtemps et je puis dire que, sur ce point, ma Société a donné l'exemple. (La Notice 1918)

\footnotetext{
${ }^{1}$ Publié en 2016 dans la revue de l'Industrie minérale et repris en 1917 chez Gauthier Villars.

2 "La notice des travaux scientifiques de M. Henri Fayol » (Fayol 1918) n'a jamais été traduite en anglais et son contenu n'a jamais été discuté, en relation avec le traité, à notre connaissance.
} 
Comme l'avait souligné Yves Cohen (Cohen 2003), Fayol ne pense pas ses principes administratifs hors de tout contexte. II voit sa doctrine comme une réponse aux nouveaux rapports entre science, industrie et travail qui s'installent à la fin du 19è siècle, et notamment au constat qui oriente toute son œuvre : la nécessité d'un nouveau type d'autorité, celle du "chef d'entreprise " dont il s'efforce de décrire, dans son traité, les missions et les compétences.

\section{Fayol, un dirigeant, un savant et un innovateur}

Fayol voulait donc être lu à partir de son expérience singulière, où l'action du dirigeant, les travaux du savant et les audaces de l'innovateur ont été indissociables. Quand il publie son Traité, il est au soir d'une carrière exceptionnelle. II a développé un groupe métallurgique et minier, dont il devient directeur général en 1888. Surtout, ce développement a été favorisé par des découvertes scientifiques et des innovations techniques parfois majeures ${ }^{3}$. La plus célèbre de ces découvertes est certainement celle de l'Invar, un acier au nickel qui ne se dilate pas. Elle est obtenue en 1896, grâce à l'aide que Fayol accorde à un physicien du Bureau des poids et mesures de Genève, Charles Edouard Guillaume, qui collaborera avec ses usines durant plusieurs décennies. Cette découverte vaudra à Guillaume le prix Nobel de physique en 1920 (Cahn 2005). Elle apporte à Fayol, et bien après lui, une moisson exceptionnelle de nouveaux marchés (Lambret et al. 1996). Auparavant, Fayol avait bousculé la géologie de son temps en élaborant la "théorie des deltas" (Baudoin 2003), à laquelle il attribue l'exploitation particulièrement efficace de sa mine de Commentry. Enfin, en 1911, il nomme à la tête du laboratoire d'Imphy, Pierre Chevenard, et lui confie une mission aussi ambitieuse qu'originale lorsqu'on la compare à l'activité des nouveaux laboratoires industriels qui naissent dans cette période (Chevenard 1933 ; cf. chap. Le Masson). Pierre Chevenard et son laboratoire incarneront pendant plusieurs décennies la nouvelle métallurgie de précision (Chevenard 1951 ; chap. Duffaut, chap. Le Masson).

\section{Le chef Fayolien : une figure difficile à penser, des concepts complexes}

Au terme d'un tel parcours, Fayol ne se propose pas d'exalter le "caractère» du vrai chef ou l'art de se faire obéir. Au contraire, on va le rappeler, il assigne au nouveau chef d'entreprise, des missions aussi complexes que surprenantes pour l'époque : celle de susciter un "perfectionnement" permanent et indéfini de toutes les activités de l'entreprise, celle d'agir comme un chef politique attentif à « constituer le corps social » de l'entreprise. Etonnement, la difficulté théorique

\footnotetext{
${ }^{3}$ Un document publié en 1918, «La notice des travaux scientifiques de M. Henri Fayol » (Fayol 1018) en fournit une recension complète.
} 
d'un tel projet, dans les référents doctrinaux de la fin du 19è siècle n'a pas été assez soulignée. Elles expliquent pourtant la complexité, l'abstraction, voire l'étrangeté des concepts fayoliens ${ }^{4}$. Car bien qu'il s'adresse à ses pairs, Fayol expose sa doctrine dans une langue qui n'est en rien celle des affaires. Plus surprenant, il ne fait aucune référence à l'économie politique de son temps. Ses concepts principaux - "prévoyance ", "programme d'action ", Etat-major, "inconnu ", perfectionnement, union du corps social - puisent sans conteste dans la philosophie politique et la théorie sociale du 18 è et du 19 è siècle, et situent l'ambition élevée du traité: une nouvelle pensée de la société et du gouvernement.

\section{Retrouver une lecture rigoureuse du Traité}

Une telle ambition théorique et sous la plume... d'un chef d'industrie! Le fait aurait dû inviter à une exégèse prudente. Mais, il est vrai que la complexité du texte de Fayol disparaît dans les traductions anglaises de Coubrough (Fayol 1930) et Storrs (Fayol 1947), publiées après sa mort. Elle échappe ainsi à son lectorat international $^{5}$ (Wren 2003). Pour relire Fayol, il faut donc s'en tenir au texte français et appréhender ses concepts les plus originaux à partir de la visée centrale qu'il indique lui-même: penser un chef adapté à un nouveau monde industriel où la recherche et la science modifient les missions de l'entreprise, ses modalités d'action et la structure des collectifs de travail.

A quoi se heurtait une telle visée ? Dans la première partie de cet article, nous reviendrons d'abord sur les figures du " chef " héritées du 19è siècle : le patron, l'administrateur et le gérant. Nous évoquerons ensuite le monde industriel Fayolien en insistant sur la nouvelle "fonction technique ", faites de bureaux d'études et de laboratoires qui s'impose dans les industries de pointe et rend obsolète les anciennes formes de l'autorité.

Dans la seconde partie, nous présenterons, sous ce nouvel éclairage, le système de concepts fayoliens. II témoigne d'une complexité et d'une cohérence peu soulignées jusqu'ici. On peut dire de la mission du chef d'entreprise fayolien qu'elle est à la fois créatrice et politique. La mission créatrice soutenue par l'effort de recherche, était en germe, à son époque à travers la littérature internationale (cf. Chapitre Le Masson) ${ }^{6}$ mais Fayol lui donne une signification inattendue. La

\footnotetext{
${ }^{4}$ Une comparaison avec le langage simple et technique de Frederic Taylor est ici éclairante

${ }^{5}$ Pour un tableau des concepts Fayoliens dont la complexité disparaît dans les traductions cf. Hatchuel et Ségrestin : fayol, théoricien de l'entreprise innovante, Actes du colloque « les ingénieurs des mines : entre technique et gestion ».

${ }^{6}$ Elle était aussi centrale chez Taylor, même si l'ingénieur américain limite son approche à la direction des usines.
} 
mission politique est plus surprenante. Fayol décrit un chef d'entreprise qui doit " constituer le corps social de l'entreprise »; qui est attaché aussi bien à l'intérêt général qu'à l'intérêt privé, les deux intérêts se rejoignant dans sa conception du "perfectionnement ». Enfin, contrastant avec les doctrines contemporaines, le traité n'évoque jamais le profit des actionnaires comme seule finalité du dirigeant. Or, un siècle après la parution du traité, avec l'installation d'un capitalisme mondialisé et financiarisé, on assiste à une crise - ou tout au moins, à un brouillage - de la figure du chef d'entreprise (Segrestin et al. 2012). Retrouver les fondements du tournant Fayolien pourrait contribuer à la refondation actuellement débattue de l'entreprise et de ses chefs.

\section{1è Partie. Figures de l'autorité et mutations industrielles vers 1890}

Au 19è siècle, trois figures du "chef " semblent suffirent pour la conduite des affaires privées ou des charges publiques ${ }^{7}$ : le patron-entrepreneur, l'administrateur et le gérant.

\section{Les figures du chef avant Fayol : le patron, l'administrateur et le gérant,}

Le patron-entrepreneur est la plus répandue. Héritier du marchand et du maitre artisan, il tirait son pouvoir de la maitrise d'un métier. Mais avec l'abolition des corporations, son statut d'entrepreneur-propriétaire et d'employeur s'est substitué à la légitimité du métier et encadre juridiquement son action. Les doctrines du "bon patron" ne manquent pas mais elles concernent le plus souvent sa responsabilité de "marchand": comptabilité, tenue des stocks, qualité de la production. A la fin du 19è siècle, on insiste sur le respect du code naissant du travail, qui enjoint de payer à temps ses ouvriers, de bien les traiter, de ne pas les humilier... Avec les luttes sociales, la question de la représentation syndicale, celle de la grève, détermineront une relation patron-ouvrier forgée dans la conflictualité et normée par le droit.

La figure de l'administrateur remonte à l'antiquité et renvoie au détenteur d'une charge publique. Des travaux récents ont montré que cette figure se construit à l'époque romaine, avec l'invention d'un modèle de la "bonne gestion " qui diffuse avec l'Empire (Crété 2016). Après la Renaissance, on retrouve cet héritage dans les traités du " bon administrateur ». Nommé par le Roi, la République ou La Cité, l'administrateur ne crée pas la mission qui lui est confiée et doit rapporter sur le contenu et les effets de son action à ses mandants. Cette reddition des comptes à un pouvoir supérieur marque une différence majeure avec le patron-

\footnotetext{
${ }^{7}$ non militaires.
} 
entrepreneur qui ne rapporte qu'à lui-même. En outre, l'administrateur doit incarner les valeurs de l'autorité qu'il représente. On attend de lui, le souci du bien public et de l'intérêt général aussi bien qu'un comportement intègre, honnête, et juste. La figure de l'administrateur est donc indissociable d'un cadre politique et de règles qu'il doit respecter et faire respecter. In fine, pèse sur lui une obligation : "celle de faire adhérer au souverain qui lui a donné sa mission " (Guerard de rouilly 1815).

Quant au gérant de société ou d'affaires, il connaît un développement important avec la société anonyme. Ce n'est pas nécessairement un patron de métier, mais il dispose de toutes les prérogatives du patron. II peut n'être pas lui-même un entrepreneur, ni même un propriétaire ${ }^{8}$. II représente la société comme personne morale et peut contracter en son nom les engagements qu'il juge bons. On peut voir le gérant comme un administrateur privé, qui doit rendre des comptes aux associés ou aux actionnaires, mais sans les repères qui définissaient la " bonne gestion " pour les administrateurs publics. Il lui faut avant tout conduire la société dans "l'intérêt " des associés. Enfin, si le droit lui accorde les pouvoirs les plus étendus, il ne précise en rien les compétences qui lui sont nécessaires et le contenu de son action, à l'exception des règles formelles liées à la société (comptes, assemblées, etc...). Le rôle du gérant a souvent fait l'objet de nombreux débats et litiges et on dispose de témoignages devant les tribunaux montrant l'ambiguïté de ses relations avec les commanditaires (Madol 1840).

Lorsque en 1860, Fayol débute sa carrière d'ingénieur, ces trois figures du chef semblaient universelles mais le nouveau monde industriel qui se construit bouscule leurs fondements en introduisant de nouvelles missions et de nouvelles compétences.

\section{Après 1860, une nouvelle fonction technique}

Les transformations de la production dont Fayol a été le témoin et l'acteur sont multiples, mais trois d'entre elles ont un écho direct dans le Traité.

a) Les bureaux d'études et la recherche industrielle : le machinisme du 19è siècle se distingue d'abord par l'invention de nouvelles machines énergétiques (machines à vapeur, moteurs thermiques, moteurs électriques...) dont l'usage est " générique " (Kogschagina 2014), c'est-à-dire, qu'il peut intéresser, et modifier, tous les métiers. Ce machinisme connaît aussi un rythme de régénération sans précédent qui est à la fois la cause et la conséquence d'un développement inédit du travail de conception. A côté des usines, Le bureau d'études devient le nouvel

\footnotetext{
${ }^{8}$ tout au plus lui demande-t-on d'être un associé symbolique.
} 
organe de la " fonction technique ». Entreprise indépendante ou service interne, il concentre les nouveaux ingénieurs et techniciens dans des collectifs inédits ${ }^{9}$. Ces bureaux d'études sont aussi le maillon entre l'usine et le laboratoire de recherche si important pour Fayol, qui entre aussi dans l'univers industriel à la même époque (cf. Le Masson et al). Ce sont les bureaux d'études (de la construction navale, de l'horlogerie, de l'automobile...) qui inventent de nouveaux usages et demandent des nouveaux alliages au laboratoire de Fayol. A l'inverse, ce sont eux qui expérimenteront les découvertes surprenantes de ce même laboratoire. A travers ces nouveaux organes, c'est un nouveau régime de savoir et d'action qui caractérise l'activité productive et qui rend l'avenir imprédictible.

b) L'administration problématique de la nouvelle "fonction technique »: L'activité des bureaux d'études et des laboratoires de recherche posaient de nouveaux problèmes de direction. Qui allait gouverner, et comment, ces nouvelles équipes? D'ailleurs étaient-elles gouvernables? Ne fallait-il pas simplement acheter leurs services et leurs conseils, comme on l'avait toujours fait avec les architectes? Les toutes premières compagnies anglaises de Chemins de fer avaient d'ailleurs commandé la conception des locomotives à des ingénieurs externes comme les Stephenson. II reste que dans beaucoup d'industries, la création de bureaux d'études internes et de laboratoires de recherches propres s'imposa posant la question du type d'administration adaptée à des cols blancs chargés de construire l'avenir des firmes (cf. Chap. Le Masson). Dans un ouvrage de 1907, Julien Dalemont décrit les services de la firme Brown-Boveri et la multiplicité des activités administratives liées à la fonction technique. II nous apprend aussi que ces activités avaient été finalement retirées à la fonction technique pour "relever désormais du Conseil (d'administration nda.) "(Dalémont 1907). Le développement des fonctions techniques d'études et de recherche provoquaient donc une rediscussion et une extension importante et inédite des activités du chef d'entreprise, ainsi que des fonctions commerciales.

c) Une représentation nouvelle du travail d'exécution. La fonction technique fixe la nature des produits à fabriquer et les conditions de leur fabrication. Elle peut aussi prescrire avec une grande précision le détail du travail ouvrier. Fréderic Taylor est le premier à penser cette évolution en inventant une "fonction méthodes " qui réplique le bureau d'études au niveau du travail d'atelier. De plus en plus conçu ex ante par des bureaux d'études et de méthodes, le travail ouvrier est donc contraint de s'intégrer dans un collectif de production et de suivre des règles imposées par l'entreprise. Cette évolution érode les anciens métiers dotés d'une autonomie traditionnelle dans leurs savoirs et leurs outils. L'ouvrier

\footnotetext{
${ }^{9}$ Cf. Numero special d'Entreprises et Histoire, $1, n^{\circ} 58,2010$, consacré aux bureaux d'études
} 
dépendra de plus en plus de la fonction technique qui fixe les conditions d'exercice de son activité. En réaction, et à la suite des grandes luttes sociales, l'Etat organisera la protection des personnels en transférant par exemple au chef d'entreprise la responsabilité en matière d'accidents du travail (loi de 1898), en raison même de son autorité sur la nouvelle fonction technique.

L'ensemble de ces transformations rendaient obsolètes les figures du patron, de l'administrateur et du gérant. II revint à Fayol de prendre conscience de ce vide doctrinal et de proposer une nouvelle "doctrine administrative». La notion évoque encore les anciens traités du " bon administrateur " (Guerard de rouilly 1815), mais on va voir, que Fayol subvertit la signification classique de la fonction administrative.

\section{2è partie. Le modèle Fayolien : la mission créative et politique du chef d'entreprise}

\section{La mission centrale : "le perfectionnement».}

Indéniablement, le chef d'entreprise Fayolien se distingue des anciennes figures du chef parce qu'il veut réussir la collaboration de la science avec le monde des affaires. Cette idée est explicitement énoncée dans la "Notice de travaux scientifiques » que Fayol publie en 1918 (Fayol 1918) où il affirme qu'il s'agit d'un message important de son Traité. Un tel objectif ancre Fayol dans son époque et limite l'universalité de son traité. Mais en quoi cette idée redéfinit-elle la mission du chef d'entreprise ?

L'appel à des expertises spéciales entrait dans le répertoire d'actions du patron, de l'administrateur ou du gérant. Mais Fayol ne fait pas de la collaboration entre la science et le monde des affaires, une action opportuniste dépendant des compétences du marché. II prend la science, comme le moteur principal d'un projet plus général, celui du "perfectionnement " qu'il désigne comme une mission majeure du dirigeant.

"Parmi ces obligations [celles du chef d'entreprise nda.] I'une des plus importantes est la recherche des perfectionnements. On sait bien qu'une entreprise qui ne progresse pas est bientôt en retard sur ses rivales et qu'il faut, par conséquent, poursuivre sans cesse le progrès dans tous les domaines (Fayol 1917 p. 78).

Ainsi Fayol accepte-t-il la concurrence, mais il en retient l'effet positif sur le progrès général. Or celui-ci, exige que tout chef d'entreprise soit investi d'une mission de perfectionnement permanent, seul gage de la pérennité de l'entreprise. En outre, Fayol ne relie pas cette mission au mandat qu'il a reçu des actionnaires. Il en fait une responsabilité intrinsèque du nouveau chef 
d'entreprise, responsabilité qui découle de sa conception des rapports entre intérêt privé et intérêt général : en perfectionnant son entreprise par la science, le chef d'entreprise accomplit son devoir vis-à-vis de l'intérêt général et protège aussi les intérêts privés, comme un capitaine de bateau protège la sécurité de ses passagers au nom de sa mission professionnelle et de l'intérêt général.

En choisissant, le terme de "perfectionnement " Fayol s'inscrit directement dans la lignée des philosophes des lumières. Depuis le 18è siècle, le terme désigne avec Condorcet, Comte et bien d'autres, la conquête des plus nobles idéaux de I'humanité et vaut pour toutes les sphères du progrès : connaissance, arts, morale. Beaucoup d'établissements d'enseignements ont, à l'époque de Fayol, un conseil dit de "perfectionnement ". Le chef d'entreprise Fayolien situe donc son autorité et sa responsabilité au plus haut niveau politique possible : contribuer au progrès collectif.

\section{Les moyens du perfectionnement : un art collectif du programme dans l'inconnu}

Mais comment le Chef d'entreprise peut-il obtenir ces perfectionnements ? A cet effet, Fayol décrit son action à l'aide d'une série de concepts tout à fait originaux.

L'Etat-major Fayolien: De son expérience, il retient d'abord que le chef d'entreprise ne peut obtenir les perfectionnements recherchés qu'en constituant autour de lui un état-major de spécialistes de toutes natures, à la fois internes et externes à l'entreprise. A cet Etat-major, il adjoint un laboratoire de recherches doté des moyens nécessaires et auquel il assigne une mission de "grande envergure» (Chevenard). Pour réussir l'insertion de cet état-major et du laboratoire dans la vie quotidienne de l'entreprise, un dispositif d'action plus général est nécessaire. Pour le décrire Fayol développe quatre concepts aussi complexes qu'originaux : prévoyance, programme, inconnu et union du corps social. On comprend mieux leur logique d'ensemble en partant de la notion d'inconnu.

L'inconnu, une conséquence inattendue du nouveau monde industriel: Fayol remarque que du fait même des progrès et des perfectionnements, le Chef d'entreprise du 20è siècle est confronté à un futur qu'il faut envisager comme présentant inévitablement une "part d'inconnu ». Certes, selon les cas, la part d'inconnu sera réduite ou majeure, mais tout contribue à l'augmenter y compris la propre action du chef d'entreprise, notamment parce qu'il ne sait pas où vont le mener les recherches qu'il engage lui-même.

L'idée "d'inconnu » est différente de celle " d'incertitude » qui domine dans la littérature économique et Fayol n'utilise jamais cette notion. Fayol ne précise pas 
de définition de l'inconnu ${ }^{10}$. Mais on peut aisément remarquer que les résultats de la recherche industrielle sont "inconnus" et pas seulement "incertains", comme on pourrait le dire des bénéfices de l'année. Dans ce second cas, le bénéfice peut prendre des valeurs variées, aléatoires et même non probabilisables, mais la notion de bénéfice restera inchangée. Alors que la recherche peut aboutir à changer la définition des notions, ou à la découverte d'objets inconnus comme ce fut le cas avec l'invar. En pratique, la distinction entre inconnu et incertitude a d'importantes conséquences. Un dirigeant peut lutter contre certaines incertitudes connues même si elles sont non probabilisables. Mais face à l'inconnu, il doit choisir entre ne rien faire et s'engager lui-même dans la recherche. Il peut aussi préparer le corps social de l'entreprise à l'inconnu, par exemple en le formant à la démarche scientifique et en favorisant les initiatives.

Pour décrire l'instrument de direction qui doit s'adapter au niveau d'inconnu et mobiliser le corps social de l'entreprise, Fayol choisit, à nouveau, un terme surprenant: "le programme général d'action ». Et, selon l'ampleur de l'inconnu, Ce programme peut prendre trois formes que l'on peut illustrer par des pratiques de Fayol :

- une liste d'actions et d'objectifs précis à atteindre lorsque « la part d'inconnu est réduite ». Ce sont par exemple les tableaux de prévisions quantitatives qu'il donne en exemple dans son traité.

- une "directive » lorsque le dirigeant ne peut indiquer qu'une orientation, sans pouvoir en dire plus, mais celle-ci peut se révéler déterminante. C'est le cas de la directive qu'il donne en 1911 à Pierre Chevenard lorsqu'il le nomme directeur du laboratoire d'Imphy (cf. article Le Masson)

- une " aventure " lorsque l'inconnu est majeur. On retrouve ici son partenariat avec Guillaume, qu'il a soutenu très tôt sans que rien ne puisse prédire ce qui allait en résulter.

Programme, commandement et corps social : Le terme de " programme " renvoie aussi au langage de l'action politique. Fayol n'utilise jamais les termes de "plan » ou de "plan prévisionnel ». Avec le terme de "Programme ", Fayol confère au chef d'entreprise une stature volontariste et visionnaire. Un programme n'est pas une réponse passive aux évènements, il exprime des convictions et un futur souhaité que l'on devra activement préparé. "Programme" évoque peu la relation entre commandement et subordonnés, il appelle plutôt l'adhésion de ceux à qui il est proposé.

\footnotetext{
${ }^{10}$ Cette notion joue un rôle central dans la théorie contemporaine de la conception. Cf. Le Masson et al. 2013
} 
D'ailleurs Fayol définit le commandement par une image inédite, "commander, c'est-à-dire faire fonctionner le personnel » (Fayol 1917 p.5.). "Fonctionner » se dit en général d'une machine, l'image pourrait donc passer pour méprisante et inhumaine. Mais Fayol évite ainsi le lieu commun : Commander, c'est se faire obéir ". "Fonctionner " ouvre un large espace d'associations qui invitent à recherche un consentement préparé, et toujours conditionnel du personnel. En effet, une machine ne fonctionne que si on en a pris soin, si on respecte des règles précises de marche, si on réagit aux signes d'alerte etc... Tous les passages du traité relatifs au personnel confirment que Fayol explore une conception du commandement qui ne fait pas de l'obéissance le seul but recherché. Fayol sait qu'il a besoin d'un assentiment du personnel pour maintenir ses objectifs de " perfectionnements » : il lui faut un personnel compétent, préparé et adapté à la variété nécessaire des programmes à lancer. En retour, un tel personnel attend que le chef d'entreprise défende avant tout la pérennité de l'entreprise, ce que Fayol appelle être un chef « prévoyant ».

\section{La prévoyance selon Fayol : une rationalité responsable face à l'inconnu}

Fayol vit dans un monde marchand et concurrentiel, mais en tant que Chef d'entreprise, la rationalité du marchand ne lui suffit plus. Elle ne permet ni de penser le futur comme un savant, ni de construire des programmes, ni de faire fonctionner le personnel. On peut donc lire le Traité comme l'exposé implicite d'une forme nouvelle de rationalité inséparable d'une théorie de la responsabilité (Hatchuel 2011).

Cette nouvelle rationalité naît d'un double constat: i) une part importante du futur est imprédictible; ii) une part du futur ne peut exister que si elle est provoquée par l'action humaine, et notamment la recherche scientifique. II en découle que le dirigeant Fayolien n'a pas pour seul but de réduire l'incertitude. Lui importe autant, de régénérer le connu, donc de faire advenir de nouveaux futurs.

Pour exprimer une idée aussi nouvelle et aussi complexe, Fayol détourne à nouveau la langue habituelle et joue avec les multiples significations de mots connus. "Prévoyance » est celui qu'il retient pour désigner cette nouvelle façon de penser les rapports entre l'entreprise et le futur. Avec ce choix, Fayol n'hésites pas à s'éloigner d'abord du sens commun. Car il désigne par prévoyance, non la capacité à prédire l'avenir, mais la capacité à agir alors même que le futur est imprédictible, et que la science tend à renforcer cette imprédictibilité.

De ce fait, l'action du dirigeant est conçue comme une assurance collective dont on attend qu'elle prémunisse contre les dangers connus et qu'elle garantisse la régénération des forces disponibles pour des combats futurs, encore inconnus. 
Avec ce détour, on se rapproche du sens traditionnel de prévoyance. Fayol nous dit d'ailleurs que "les français sont prévoyants mais que leur gouvernement ne l'est pas ", signifiant ainsi que le régime d'action de l'Etat ne correspond pas au modèle fayolien Enfin, Fayol ne pouvait ignorer que prévoyance signifiait aussi entraide et solidarité, ni que le mot était inséparable de la justice sociale et de la cohésion nationale. Nul surprise donc quand il affirme que le chef d'entreprise doit réaliser « la constitution du corps social » (P.4), et « l'union du personnel » ( $p$. $5)$, car ce sont là des formes de prévoyance indispensables au bon fonctionnement de son programme d'action.

Avec ces notions, on est loin du vocabulaire classique d'un gérant et d'un patron, et a fortiori d'un marchand. On est plus proche de la figure de l'administrateur public qui n'oublie pas que son action participe de la construction d'un espace politique.

Le chef d'entreprise Fayolien construit ainsi une figure inédite qui :

- étend considérablement l'action du patron et du gérant en introduisant l'inconnu, la science et la recherche de perfectionnement

- importe dans la sphère privée, une théorie politique de l'action et de la constitution du corps social.

On voit comment Fayol introduit une rationalité responsable qui nous est aujourd'hui familière: nous attendons que les entreprises innovent, qu'elles investissent dans la recherche, dans de nouveaux produits, dans de nouvelles compétences. Mais avant Fayol, rien n'assignait ce type de tache à un patron, un administrateur ou un gérant d'affaires. Fayol aboutit aussi implicitement à une critique nouvelle et profonde de la rationalité de l'homo economicus, car celle-ci se révèle inopérante face au nouveau type d'inconnu créé par la science.

In fine, Fayol invitait, sans le dire, à considérer l'entreprise issue du nouveau monde industriel, comme un nouveau type d'entité collective, qui transforme simultanément les fondements du savoir, de l'économique, du social et du travail. Cette nouvelle entité ne pouvait être gouvernée que selon une conception réinventée du chef et de la bonne administration.

\section{Conclusion: Fayol, théoricien d'un modèle créatif/politique du chef d'entreprise}

Fayol a trop souvent été lu sans discussion préalable sur l'appareil critique, linguistique et interprétatif nécessaire à son étude. C'était oublier que Fayol était 
un savant renommé et un innovateur remarquable. C'était négliger aussi le contexte de son œuvre et l'ambition théorique qui sous-tendait son projet. Notre relecture confirme cette ambition et l'ampleur des moyens conceptuels mobilisés par Fayol. La mise à jour de cet édifice intellectuel est porteuse de multiples enseignements.

a. La théorie administrative Fayolienne n'est pas une somme de recettes universelles, hors du temps. Elle prend acte, d'un tournant historique majeur: la formation de nouveaux rapports entre science, industrie et travail. Ces nouveaux rapports inaugurent un régime inédit de renouvellement des richesses. Ils favorisent aussi de nouveaux collectifs, dont la nature et le gouvernement sont problématiques. En théorisant un nouveau type d'autorité, Fayol contribue à la domestication de ces nouveaux collectifs, il permet la formation d'une nouvelle épistémè (au sens de Foucault), celui du "chef d'entreprise moderne ", qui rend visible, l'entreprise moderne elle-même. Cette dernière n'est plus l'ancienne compagnie, elle n'est pas non plus assimilable à une organisation bien réglée comme les administrations d'Etat.

b. La théorie Fayolienne ne doit rien à la théorie économique, pas même à celle de l'entrepreneur. L'absence des notions économiques les plus élémentaires (profit, marchés, capital) ${ }^{11}$ aurait dû être mieux soulignée. Car, on peut difficilement accuser Fayol de ne pas connaître la réalité de la vie des affaires. II faut donc prendre cette absence au sérieux. Ce point mériterait une plus longue discussion mais on peut retenir, au terme de cette étude, deux raisons qui éloignent Fayol de la théorie économique.

Fayol considère que la source majeure de la création de la richesse, n'est ni dans le capital, ni dans le travail, ni dans les moyens de production, mais dans la recherche scientifique et dans le perfectionnement permanent. Fayol invite donc à penser une rationalité créatrice, distincte de la rationalité fins-moyens de la pensée économique, qu'il s'efforce de décrire avec les concepts de prévoyance, $d^{\prime}$ inconnu, et de programme ${ }^{12}$.

Cette rationalité créatrice ne peut se déployer que dans de nouveaux systèmes collectifs de travail dont il faut inventer le mode de gouvernement. Fayol ne remet en cause ni l'économie de marché, ni le contrat de travail, il reconnait même l'intérêt des incitations économiques. Mais les organisations héritées du monde marchand ancien sont à ses yeux incapables de réaliser "les programmes d'action " notamment à "long terme », dans l'inconnu qu'exige le nouveau

\footnotetext{
${ }^{11}$ En dehors d'un tableau comptable sommaire p.54-55.

${ }^{12}$ II n'hésite pas à comparer le chef d'entreprise à un Architecte lorsqu'il s'agit de concevoir le programme ;
} 
monde industriel. Il faut donc inventer de nouveaux liens sociaux, et cette tâche incombe aussi à ce nouvel acteur politique qu'est le chef d'entreprise. C'est sur ce point qu'il prend appui dans la philosophie politique de son temps et dans l'héritage des lumières. II y trouve le projet général de perfectionnement qui constitue la promesse collective sur laquelle une société peut se constituer et s'unir.

c. L'inventivité théorique de Fayol a été sous-estimée. Pour mieux apprécier la portée des concepts fayoliens on peut les rapprocher de deux autres auteurs, Franck Knight et Max weber, qui sont ses contemporains et s'efforçaient aussi de penser les transformations du monde industriel de la période.

L'économiste Franck Knight (Knight 1921) repense la nature de l'incertitude dans le fonctionnement économique. II reconnaît l'existence d'une incertitude non probabilisable qui invalide la théorie classique des marchés. Knight en conclut que le fonctionnement économique devient dépendant de " managers " qui ont pour fonction de prendre des décisions en situation d'incertitude radicale. La valeur économique de ces managers réside alors, selon Knight, dans la réduction des incertitudes qu'ils réalisent pour l'économie dans son ensemble. Néanmoins Knight ne réussit pas à justifier l'investissement dans la recherche, au niveau d'une entreprise, et il ne propose aucune théorie du fonctionnement de l'entreprise : celle-ci reste une simple réunion de travail et de capital.

Max Weber pense l'émergence de l'organisation économique notamment à travers la théorie dite rationnel/légale. Dans ce modèle, la rationalité prédominante est instrumentale (adaptation des moyens aux fins) et la vie sociale est construite sur l'impersonnalité des lois et des règles. Ces deux caractéristiques décrivent des organisations de type bureaucratiques ou technocratiques.

Le modèle fayolien se confond-il avec le modèle Weberien? Une lecture trop hâtive du traité a pu laisser penser que Fayol décrit une bureaucratie. Mais Fayol indiquait lui-même qu'il s'opposait au type d'administration qui domine dans les organismes publics parce qu'elle n'avait pas de programme d'action et pas de prévoyance. Par ailleurs, dans le modèle Fayolien, la constitution du corps social n'est pas le résultat de règles impersonnelles, seraient-elles source de justice, mais exige aussi le perfectionnement collectif et individuel. Enfin, l'entreprise Fayolienne n'est pas une technocratie parce que l'autorité n'y est pas détenue par la fonction technique. Ce point est au cœur du modèle Fayolien puisqu'il vise précisément à inventer un gouvernement de la fonction technique et de la recherche, au même titre que pour les autres fonctions de l'entreprise. 
Une conclusion s'impose: Fayol aboutit à un modèle que nous proposons d'appeler créatif/politique, par comparaison avec le modèle rationnel/légal de max Weber. Ce modèle se révèle à l'analyse plus adapté au nouveau monde industriel, scientifique et innovateur, que les théories de Knight et de Weber qui ont eu portant une réception scientifique beaucoup plus large que Fayol.

Avec le recul de l'histoire, la confrontation à ces deux auteurs permet de cerner la logique profonde de la découverte Fayolienne. Fayol n'est prisonnier ni du rationalisme économique d'un Knight, ni du structuralisme sociologique de max Weber. Son modèle prend ses racines dans l'idée que la quête permanente de progrès et le développement continu des sciences, plonge les sociétés modernes dans un univers où le futur est doublement inconnu, il est inconnu du fait des autres, mais il est aussi inconnu du fait de soi. Dans cet univers, la rationalité des décisions n'a plus de socle épistémologique assuré : compte surtout la capacité à mobiliser pour inventer et à découvrir, ou réciproquement, la capacité à inventer et découvrir pour mobiliser. Le chef d'entreprise devient le capitaine d'une expédition dans une contrée largement inexplorée. Dans une telle expédition, la cohésion sociale joue un double rôle, elle aide à construire le programme d'action en mobilisant les connaissances de tous, mais elle aide aussi à le mettre en œuvre une fois qu'il est fixé. Fayol aurait donc été le premier penseur à comprendre que la dynamique des entreprises annonçait la fin des théories classiques de l'économie et du système social, pour le meilleur et pour le pire.

\section{Et aujourd'hui ?}

Le capitalisme financier a provoqué une étrange régression anti-fayolienne dans un monde qui n'a jamais été aussi fayolien. La dimension créative n'a jamais été aussi nécessaire et présente dans la compétition contemporaine par l'innovation. De même que la dimension politique de l'action du chef d'entreprise s'étend à un " corps social " qui va au-delà des personnels et des actionnaires, et inclut des partenaires, des territoires, des Etats, et des milieux écologiques. Le concept de perfectionnement est passé de l'histoire humaine à celle de la planète.

A l'inverse, financiers et codes de gouvernement d'entreprise (Corporate Governance), ont favorisé depuis trois décennies, le retour d'une néo-gérance, exacerbant l'alignement du dirigeant sur les seuls intérêts des actionnaires. Les effets corrosifs et destructeurs de cette régression sont aujourd'hui patents. On observe un divorce croissant entre entreprise et société (au sens des associés) mais aussi entre les entreprises et les Etats (Segrestin et al. 2012). Retrouver la pensée fayolienne, ce n'est pas appeler à une illusoire restauration, un siècle après, du type de dirigeant qui fut le sien. Mais la valeur du modèle Fayolien tient 
à ce qu'il offre une alternative théorique oubliée aux erreurs du rationalisme économique, et à la rigidité des structuralismes sociaux. II permet de penser le gouvernement des actions collectives créatrices. Comme beaucoup de grandes pensées du passé, il offre des pistes pour l'avenir.

\section{References}

Beaudoin, B. 2003. Henri Fayol, géologue perspicace et novateur? In J.-L. Peaucelle (Ed.), Henri Fayol, Inventeur des outils de gestion: 47-67. Paris: Economica.

Cahn, R. W. 2005. An unusual Nobel prize. Notes and Records of the Royal Society, 59(2): 145-153.

Chambers, P. 1974. Europe's greatest management pioneer. International management, 29: 48-51.

Chevenard, P. 1933. L'installation et l'organisation d'un laboratoire sidérurgique moderne. Mémoires de la société des ingénieurs civils de France, septembreoctobre: 3-52.

Chevenard, P. 1951. La recherche scientifique dans l'industrie française. Réflexions et souvenirs. Mémoires de la société des ingénieurs civils de France, Janvier-Avril.

Cohen, Y. 2003. Fayol, un instituteur de l'ordre industriel. Entreprises et Histoire, 34(3): 29-67.

Crété M., "la gestion à l'époque moderne. La naissance d'un nouveau modèle d'action publique" (A paraître 2016).

Dalémont, Julien. (1907). La construction des machines électriques, Librairie polytechnique Ch. Béranger, Paris.

Guerard de Rouilly,A. (Baron), Principes généraux d'administration, Favre Paris 1815.

Fayol, H. 1918. Notice sur les travaux scientifiques et techniques de M. Henri Fayol. Paris: Gauthier-Villars et Cie Editeurs. http://gallica.bnf.fr/ark:/12148/bpt6k904289.r=notice+fayol.langFR.

Fayol, H. 1930. Industrial and General Administration (J. A. Coubrough, Trans.). London: Sir Isaac Pitman \& Sons.

Fayol, H. 1949. General and Industrial Management (C. Storrs, Trans.). London: Sir Isaac Pitman \& Sons. 
Fells, M. J. 2000. Fayol stands the test of time. Journal of Management History, 6(2000): 345-360.

Hatchuel A., 2011, "Aux origines de la Gestion scientifique" in "Saussois J.M., "Les organisations" Sciences Humaines 2011.

Knight, F. H. 1921. Risk, Uncertainty, and Profit. New York: Sentry Press.

Kokshagina, O., Risk management in double unknown : theory, model and organization for the design of generic technologies, Thèse Mines-ParisTech/ PSL 2014.

Lambret, E., \& Saindrenan, G. 1996. The discovery of Invar and the metallurgical works of Charles-Edouard Guillaume. In J. Wittenauer (Ed.), The invar effect: a centennial symposium: 39-47. Warrendale, PA: The Minerals, Metals and Materials Society.

Lamond, D. 2004. A matter of style: reconciling Henri and Henry. Management Decision, 42(2): 330-356.

Madol G., Compte rendu d'un gérant à ses commanditaires, 1840 (document Gallica).

Mintzberg, H. 1975. The manager's job: folklore and fact. Harvard Business Review, 53(July-August 1975): 49-61.

Parker, L. D., \& Ritson, P. A. 2005. Revisiting Fayol: Anticipating Contemporary Management. British Journal of Management, 16(3): 175-194.

Peaucelle, J.-L. 2003a. Fayol méconnu et toujours original. Entreprises et Histoire, 34(3): 5-7.

Peaucelle, J. L. 2003b. Henri Fayol: inventeur des outils de gestion: textes originaux et recherches actuelles: Economica.

Pryor, M. G., \& Taneja, S. 2010. Henri Fayol, practitioner and theoretician-revered and reviled. Journal of Management History, 16(4): 489-503.

Reid, D. 1995a. Fayol: from experience to theory. Journal of Management History (Archive), 1(3): 21-36.

Reid, D. 1995b. Reading Fayol with 3D glasses. Journal of Management History (Archive), 1(3): 63-71.

Urwick, L. 1949. Foreword. In H. Fayol (Ed.), General and Industrial Management. London: Pitman. 
Weber, M. 1922. Wirtschaft und Gesellschaft [Economy and Society]. Tübingen: J. C. B. Mohr (Paul Siebeck).

Wren, D. A. 1992. The nature of managerial work: A comparison of Real Managers and traditional management. Journal of Managerial Issues, 4: 17-30.

Wren, D. A. 1995. Henri Fayol: learning from experience. Journal of Management History (Archive), 1(3): 5-12.

Wren, D. A. 2001. Henri Fayol as strategist: a nineteenth century corporate turnaround. Management Decision, 39(6): 475-487.

Wren, D. A. 2003. The influence of Henri Fayol on management theory and education in North America. Entreprises et histoire(3): 98-107.

Wren, D. A., Bedeian, A. G., \& Breeze, J. D. 2002. The foundations of Henri Fayol's administrative theory. Management Decision, 40(9): 906-918.

Yoo, J. W., Lemak, D. J., \& Choi, Y. 2006. Principles of management and competitive strategies: using Fayol to implement Porter. Journal of Management History, 12(4): 352-368. 